\title{
Qualidade de ninho, ganho de peso dos láparos e custo do uso da maravalha como substrato de nidificação de coelhas
}

\author{
Nest quality, weight gain of kits and cost of using wood shavings as a \\ nesting substrate for rabbits
}

\section{Calidad del nido, ganancia de peso de la camada y costo del uso de virutas de madera como sustrato de anidación para conejos}

\author{
Kassy Gomes da Silva ${ }^{1}$, Isabella Rotta ${ }^{2}$, Leandro Batista Costa $^{3}$, Cristina Santos \\ Sotomaior $^{3}$ \\ ${ }^{1}$ Discente do Programa de Pós-graduação em Ciência Animal - Pontifícia Universidade Católica do \\ Paraná, campus Curitiba, Brasil; \\ ${ }^{2}$ Discente do curso de Medicina Veterinária - Pontifícia Universidade Católica do Paraná, campus \\ Curitiba, Brasil \\ ${ }^{3}$ Docente do Programa de Pós-graduação em Ciência Animal - Pontifícia Universidade Católica do \\ Paraná, campus Curitiba, Brasil. *E-mail: cristina.sotomaior@ pucpr.br
}

\begin{abstract}
RESUMO
O fornecimento de ninho com substrato é importante como medida de manejo pré-parto da coelha gestante. Diversos materiais são utilizados como substrato, variando com a disponibilidade na região. Além de ser acessível ao produtor, o substrato precisa atender às necessidades de bem-estar animal, tanto da coelha quanto da ninhada. O objetivo do presente estudo foi avaliar a maravalha como alternativa ao feno como material de nidificação para coelhas, quanto aos índices zootécnicos dos láparos, qualidade de ninho e custo-benefício ao produtor. O experimento foi conduzido com 24 coelhas Nova Zelândia Branco, separadas em dois tratamentos, com 12 repetições cada, sendo eles: feno (controle) e maravalha. Os dados avaliados foram: qualidade de ninho [pré-parto (QNPRE) e pós-parto (QNPOS)], ganho de peso diário (GPD) e mortalidade dos láparos e custo-benefício ( $\mathrm{R}$ / ninho e $\mathrm{R}$ / láparo desmamado). Não houve diferença significativa para os parâmetros de QNPRE, QNPOS, GPD e mortalidade, do nascimento ao desmame, entre os tratamentos. Não houve correlação entre QNPOS e mortalidade entre o $2^{\circ}$ e $10^{\circ}$ dia de vida. A maravalha apresentou maior custo por ninho e por láparo desmamado. A maravalha pode ser utilizada como substrato de nidificação para coelhas, porém o acesso e o custo regional dos substratos podem ser utilizados pelo produtor como determinantes da escolha do material a ser utilizado na granja.
\end{abstract}

Palavras-chave: feno, láparo, ninho, Oryctolagus cuniculus 


\begin{abstract}
The provision of nest with substrate is important as a measure of pre-birth management of the pregnant rabbit. Several materials are used as substrate, varying with availability in the region. Besides to be accessible to the farmer, the substrate must fulfill the animal welfare needs of the doe and litter. The objective of the present study was to evaluate wood shavings as an alternative to hay as nesting material for rabbits, in relation to the zootechnical data of the litter, nest quality and cost-benefit to the farmer. The experiment was carried out with 24 New Zealand White does, separated in two treatments, with 12 repetitions each: hay and wood shavings. The evaluated data were nest quality [prepartum (QNPRE) and post-partum (QNPOS)], daily weight gain (DWG) and mortality of the litter and cost-benefit ( $\mathrm{R} \$ /$ nest and $\mathrm{R} \$$ / weaned kit). There was no significant difference for the parameters of QNPRE, QNPOS, DWG and mortality, from birth to weaning, between treatments. There was no correlation between QNPOS and mortality between the 2nd and 10th day of life. Wood shavings were more expensive per nest and per weaned kit. The wood shavings can be used as nesting substrates for rabbits; however, the availability and the regional cost of the substrates can be used by the farmer for the choice of material to be used on the farm.
\end{abstract}

Key words: hay, kit, nest, Oryctolagus cuniculus

\title{
RESUMEN
}

La provisión de nido con sustrato es importante como medida del manejo prenatal de la coneja preñada. Se usan muchos materiales como sustrato, que varían según la disponibilidad en la región. Además de ser accesible para el productor, el sustrato debe satisfacer las necesidades de bienestar animal tanto del conejo como de la camada. El objetivo del presente estudio fue evaluar las virutas de madera como una alternativa al heno como material de anidación para conejos, en relación con los índices zootécnicos de las letrinas, la calidad del nido y el costo-beneficio para el productor. Fueran utilizadas 24 conejas Nueva Zelanda Blancos, separadas en dos tratamientos, con 12 repeticiones cada uno, que son: heno (controle) y virutas de madera. Los datos evaluados fueron: calidad del nido [antes del parto (CNPRE) y después del parto (CNPOS)], ganancia de peso diario (GPD) y mortalidad de la camada y costo-beneficio (R\$ / nido y $\mathrm{R} \$$ /destetado). No hubo diferencias significativas para los parámetros de CNPRE, CNPOS, GPD y mortalidad, desde el nacimiento hasta el destete, entre los tratamientos. No hubo correlación entre CNPOS y mortalidad entre el $2^{\circ}$ y $10^{\circ}$ día de vida. Las virutas de madera tuvieron el mayor costo por nido y por conejo destetado. Las virutas de madera pueden ser utilizadas como sustratos de anidación para conejos, sin embargo, la disponibilidad y el costo regional de los sustratos pueden ser utilizados por el productor como determinantes de la elección del material que se utilizará en la granja.

Palabras clave: camada, heno, nido, Oryctolagus cuniculus 


\section{Introdução}

$\mathrm{Na}$ natureza, a coelha constrói um ninho formado de matéria vegetal seca e pelos do próprio corpo, normalmente em tocas denominadas "berçário", para abrigar os láparos após o nascimento (ROSS et al., 1963; SELTMANN et al., 2017). O cunicultor deve fornecer o ambiente adequado para que a coelha possa expressar seu comportamento natural de nidificação. $\mathrm{O}$ manejo pré-parto realizado na granja inclui a disponibilização de um ninho artificial e um substrato, que a coelha poderá utilizar para a construção do ninho (FERREIRA et al., 2012). A falta de um ninho ou má qualidade deste pode resultar em perda da ninhada, pela falta de mecanismos de termorregulação eficientes nos neonatos, resultando em hipotermia e óbito dos láparos (KLINGER e TOLEDO, 2018).

Um ninho com boa qualidade terá a superfície coberta por material de nidificação (pelo + substrato), cobrindo os láparos para mantê-los seguros e aquecidos (VELLA e DONNELLY, 2012). O substrato escolhido deve ser confortável para a coelha e para os láparos. Diversos materiais são utilizados, sendo a maravalha, feno e palhas os mais comuns (OLIVEIRA et al., 2017; SZENDRO et al., 2019). Além do conforto, parâmetros como mortalidade, peso dos láparos e custo ao produtor também precisam ser considerados (SZENDRO et al., 2019).

No Brasil, o alto custo de produção é um dos fatores limitantes da produção de coelhos de carne (MACHADO e FERREIRA, 2014). Assim, a escolha de um material de nidificação com baixo custo, com manutenção do bem-estar animal e dos índices produtivos dos coelhos é importante para contribuir na diminuição do custo da produção brasileira.

Diante disso, este estudo foi realizado para avaliar a maravalha, uma alternativa ao feno, como material de nidificação para coelhas em relação à qualidade de ninho, desempenho zootécnico dos láparos e custo-benefício.

\section{Material e Métodos}

O estudo foi realizado no setor de cunicultura da Fazenda Experimental Gralha Azul (FEGA), da Pontifícia Universidade Católica do Paraná, em Fazenda Rio Grande, Paraná, Brasil, no período de agosto de 2019 a março de 2020. O estudo foi aprovado pelo Comitê de Ética no Uso de Animais (CEUA)PUCPR, sob protocolo de número 01640. 
Foram utilizadas 24 coelhas adultas da raça Nova Zelândia Branco, alojadas de forma individual em gaiolas suspensas de arame com $80 \times 60 \times 45$ $\mathrm{cm}$, com fornecimento de ração e água à vontade. Feno de tifton à vontade foi fornecido após o nascimento dos láparos, para evitar interferência do uso desse feno na preparação do ninho pela coelha. As condições ambientais foram de luz natural e uso de cortinas para controle de temperatura e ventilação. $\mathrm{O}$ manejo reprodutivo foi por monta natural, com o diagnóstico de gestação aos 14 dias pósmonta por meio de palpação abdominal.

Foram testados dois tratamentos, o uso do feno de tifton (grupo controle) e da maravalha, com 12 animais em cada tratamento, distribuídos em um delineamento inteiramente casualizado (DIC), sendo a coelha a unidade experimental. Em cada gaiola, foi colocado um ninho de madeira, aberto, com $40 \mathrm{~cm}$ de comprimento $\times 23 \mathrm{~cm}$ de largura $\times 23 \mathrm{~cm} \mathrm{e} 8 \mathrm{~cm}$ de alturas máxima e mínima, respectivamente, com uma camada de, aproximadamente, $5 \mathrm{~cm}$ de altura de substrato, de acordo com o tratamento. Os ninhos foram pesados em balança digital para a anotação da quantidade de substrato utilizado antes e após a colocação do substrato e foram inseridos nas gaiolas das coelhas três dias antes do parto.

No dia 1, a avaliação da qualidade de ninho pré-parto foi realizada. Caso a coelha tenha retirado todo material do ninho, uma reposição foi feita, até alcançar novamente os $5 \mathrm{~cm}$ de substrato; caso a retirada tenha sido parcial ( $<3 \mathrm{~cm}$ presentes no ninho), foi feita uma reposição até alcançar $3 \mathrm{~cm}$ de substrato no ninho. O material reposto também foi pesado e anotado. Após a reposição no primeiro dia, nenhuma outra foi realizada até $o$ final do experimento.

No dia do nascimento (dia 0 ), o manejo de retirada de natimortos e restos placentários foi realizado, com reposição do material, se necessário, até atingir 3 $\mathrm{cm}$ de altura. Os láparos permaneceram com a coelha, para a primeira mamada. No primeiro dia pós-parto, a ninhada foi padronizada em 6 láparos por coelha; uma coelha do tratamento com maravalha permaneceu com 7 láparos. Os ninhos foram retirados 21 dias pósparto.

O experimento foi finalizado aos 35 dias de idade dos láparos, considerado o dia do desmame. Devido ao número de animais necessários para um $n$ representativo, o experimento foi repetido três vezes no tempo, com oito 
fêmeas por repetição no tempo (4 fêmeas no tratamento feno e 4 fêmeas no tratamento maravalha).

Os parâmetros avaliados foram a qualidade de ninho pré (QNPRE) e pósparto (QNPOS), temperatura/umidade do ar ambiental, ganho de peso diário (GPD) dos láparos, mortalidade dos láparos e custo-benefício. A qualidade de ninho foi avaliada no pré-parto, de acordo com descrito por Blumetto et al. (2010), e no pós-parto (dias 2 a 10), com método de Farkas et al. (2016).

$\mathrm{Na}$ avaliação de QNPRE, foi avaliada a preparação do ninho pela coelha, que consistia na observação de presença de pelo (PP), mistura do material do ninho com o pelo (MM) e preservação do material original (substrato) no ninho (PS) (Tabela 1).

Tabela 1. Parâmetros avaliados em ninhos de coelhas Nova Zelândia branco, com diferentes substratos (feno ou maravalha) um dia antes do parto

\begin{tabular}{lcccc}
\hline \multicolumn{1}{c}{ Parâmetro } & 1 & 2 & Escore & 4 \\
\hline $\begin{array}{l}\text { Presença de pelos no } \\
\text { ninho (PP) }\end{array}$ & Sem pelos & $>50 \%$ do substrato visível & $<50 \%$ do substrato visível & Só pelos visíveis \\
$\begin{array}{l}\text { Mistura de pelos com } \\
\text { substrato (MM) }\end{array}$ & Sem mistura & Nível moderado & Nível alto & - \\
$\begin{array}{l}\text { Preservação do substrato } \\
\text { no ninho (PS) }\end{array}$ & $<30 \%$ de substrato & $\begin{array}{c}\text { entre 30 a } 60 \% \text { de } \\
\text { substrato }\end{array}$ & $>60 \%$ de substrato & - \\
\hline
\end{tabular}

Blumetto et al. (2010)

$\mathrm{Na}$ avaliação de QNPOS foi observada a cobertura de pelos e/ou substrato sobre os filhotes (adaptado de FARKAS et al., 2016), sendo a pontuação: 1= láparos descobertos (100\% visíveis/ 0\% não visíveis); $2=$ láparos pouco cobertos $(25 \%$ não visíveis); $3=$ láparos parcialmente cobertos (50\% não visíveis); 4= láparos cobertos (75\% não visíveis); 5= láparos completamente cobertos $(100 \%$ não visíveis).
Para as mensurações de temperatura e umidade do ar ambiental foi utilizado um termohigrômetro digital (modelo 7429.02.0.00, Incoterm), sendo mensurados os parâmetros de máxima, mínima e do momento durante todo o período experimental. A partir do dia 1 de vida, as ninhadas foram pesadas semanalmente, em balança digital (Prix 3, Toledo), até os 35 dias de vida, totalizando 5 pesagens. $\mathrm{O}$ ganho de peso diário (GPD) médio da ninhada foi calculado para o período de aleitamento, 
dividindo-se o GPD total pelo número de láparos. A mortalidade dos láparos foi calculada do nascimento ao desmame, que ocorreu aos 35 dias de vida. $\mathrm{O}$ número de láparos desmamados também foi observado. O custo do uso de cada substrato foi calculado através do consumo total de material (inicial + reposto), obtendo-se o custo de substrato por ninho ( $\mathrm{R} \$ /$ ninho) e por láparo desmamado ( $\mathrm{R} \$ / \mathrm{n}^{\circ}$ de desmamados). Os preços utilizados foram $\mathrm{R} \$ 0,93 / \mathrm{kg}$ para feno e $\mathrm{R} \$ 1,00 / \mathrm{kg}$ para maravalha (cotação de maio/2020).

A análise estatística foi realizada com o uso da análise de variância (ANOVA). O fator tempo foi inserido no modelo. Não houve diferença estatística entre as repetições no tempo. Para as variáveis paramétricas (quantidade de material, custo, mortalidade, peso do láparo, ganho de peso diário, número de láparos desmamados), quando diferença entre as médias dos tratamentos foi observada $(p<0,05)$, utilizou-se teste de
Tukey para comparação entre as médias. Para variáveis não-paramétricas (presença de pelo, mistura do material do ninho com o pelo, preservação do material original no ninho e cobertura de pelos e/ou material sobre os filhotes), foram usados os testes de Kruskal-Wallis e quando $\mathrm{p}<0,05$, utilizou-se o teste de Mann-Whitney. A correlação entre as variáveis foi calculada com o teste de correlação de Pearson para variáveis paramétricas (temperatura ambiental, umidade relativa do ar e mortalidade) e o teste de correlação de Spearman para as variáveis não-paramétricas (QNPOS, mortalidade). O nível de significância utilizado foi de 5\%. O software IBM SPSS versão 25 foi utilizado para a análise estatística dos dados.

\section{Resultados e Discussão}

Não houve diferença significativa $(\mathrm{P}>0,05)$ para a $\mathrm{QNPRE}$ (considerando PP, MM e PS) entre os substratos utilizados (Tabela 2).

Tabela 2. Escores de qualidade de ninho pré-parto (\%) de coelhas Nova Zelândia Branco, de acordo substrato utilizado no ninho.

\begin{tabular}{cccccc}
\hline \multirow{2}{*}{ Parâmetro } & Material & \multicolumn{4}{c}{ Escore (em \% de ninhos) } \\
\cline { 3 - 5 } & feno & 75,0 & 16,7 & 3 & 4 \\
\hline \multirow{2}{*}{ PP } & maravalha & 83,3 & 8,3 & 0,0 & 8,3 \\
& feno & 91,7 & 0,0 & 8,3 & 0,0 \\
\multirow{2}{*}{ PS } & maravalha & 75,0 & 16,7 & 8,3 & 0,0 \\
& feno & 33,3 & 41,7 & 8,3 & 0,0 \\
& maravalha & 8,3 & 66,7 & 25,0 & 0,0 \\
& m & & & 25,0 & 0,0 \\
\hline
\end{tabular}

PP: presença de pelo; MM: mistura do material do ninho com o pelo; PS: preservação do material original (substrato) no ninho. 
Foi observado que a QNPRE para ambos substratos apresentou escores baixos (1 e 2) para PP, MM e PS um dia antes do parto. A ausência de pelos na maioria dos ninhos pode ser explicado pelo momento escolhido para a avaliação (1 dia antes do parto), pois a coelha ainda poderia preparar o ninho nas 24 horas seguintes, incluindo a colocação de pelos. A preparação do ninho, com a mistura dos pelos no substrato, pode ocorrer desde alguns dias, horas ou imediatamente antes do parto (ZARROW et al., 1961; GONZÁLEZ-MARISCAL et al., 2016). O MM foi diretamente influenciado pelo PP, já que a ausência dos pelos leva o escore do MM automaticamente para 1 (sem mistura). No caso da PS, o consumo de feno pelas coelhas do grupo controle e a exploração da maravalha (comportamento de escavar e consequente retirada de maravalha do ninho) pelas coelhas podem ter influenciado na avaliação deste parâmetro. Oliveira et al. (2017) observaram que o PS foi maior para ninhos com maravalha do que com feno; uma possível explicação é o momento de avaliação, pois as avaliações foram realizadas em dois momentos no mesmo dia, sendo possível que a última avaliação tenha sido realizada mais próximo ao momento do parto do que no estudo atual, onde a avaliação foi única e 1 dia antes do parto. A QNPRE similar entre os substratos também foi observada por Blumetto et al. (2010).

Não houve diferença significativa $(p>0,05)$ entre a QNPOS e os substratos utilizados (Tabela 3). Os resultados foram contrários aos observados por Farkas et al. (2016) que relataram melhor qualidade de ninho com o uso de feno do que com maravalha no $5^{\circ}$ dia pós-parto. O tamanho amostral do atual trabalho pode não ter sido suficiente para confirmar os resultados de Farkas et al. (2016).

Tabela 3. Escores de qualidade de ninho pós-parto (cobertura de pelos, \%) de coelhas Nova Zelândia Branco, de acordo substrato utilizado no ninho

\begin{tabular}{cccccc}
\hline \multirow{2}{*}{ Material } & \multicolumn{5}{c}{ Escore (em \% de ninhos) } \\
\cline { 2 - 5 } & 1 & 2 & 3 & 4 & 5 \\
\hline Feno & 16,7 & 14,8 & 22,2 & 21,3 & 25,0 \\
Maravalha & 25,9 & 19,5 & 23,1 & 17,6 & 13,9 \\
\hline
\end{tabular}


O GPD não apresentou diferença significativa quanto ao substrato (Tabela 4), resultado também relatado por OLIVEIRA et al. (2017) quando comparado feno, maravalha e tiras de jornal. Não houve diferença significativa $(p>0,05)$ entre os tratamentos para o peso individual dos láparos. A falta de relação entre o peso dos láparos e o substrato de nidificação foi descrito por BLUMETTO et al. (2010), FARKAS et al. (2016) e OLIVEIRA et al. (2017) quando estudaram palha, jornal em tiras, fibra de madeira $\left(\right.$ Lignoce $\left.{ }^{\circledR}\right)$, bem como feno e maravalha.

Tabela 4. Ganho de peso diário (GPD, média \pm desvio padrão, g) do Nascimento ao desmame e peso aos 7, 14, 21, 28 e 35 dias de vida dos láparos, de acordo com o substrato

\begin{tabular}{lcc}
\hline \multicolumn{1}{c}{ Parâmetros } & & Substrato \\
& Maravalha & Feno \\
\hline GPD $(\mathrm{g})$ & $24,8 \pm 5$ & $27,2 \pm 4$ \\
Peso médio dos láparos & & \\
Dia 1 $(\mathrm{g})$ & $55,6 \pm 7(\mathrm{n}=73)$ & $54,5 \pm 8(\mathrm{n}=72)$ \\
Dia $7(\mathrm{~g})$ & $125,8 \pm 55(\mathrm{n}=65)$ & $144,2 \pm 32(\mathrm{n}=60)$ \\
Dia 14 $(\mathrm{g})$ & $254,3 \pm 61(\mathrm{n}=64)$ & $295,8 \pm 60(\mathrm{n}=56)$ \\
Dia 21 $(\mathrm{g})$ & $390,8 \pm 123(\mathrm{n}=62)$ & $405,0 \pm 86(\mathrm{n}=55)$ \\
Dia 28 $(\mathrm{g})$ & $580,8 \pm 134(\mathrm{n}=62)$ & $615,8 \pm 102(\mathrm{n}=55)$ \\
Dia 35 (desmame, $\mathrm{g})$ & $903,3 \pm 161(\mathrm{n}=62)$ & $978,3 \pm 134(\mathrm{n}=55)$ \\
\hline
\end{tabular}

O número médio de láparos desmamados foi de 4,9 \pm 1,2 láparo, sem diferença significativa $(\mathrm{p}>0,05)$ entre a média de láparos desmamados do tratamento com maravalha $(5,2 \pm 1,1$ láparos) e com feno (4,6 \pm 1,2 láparos). A mortalidade de láparos durante todo período experimental foi de 19\%. A mortalidade na primeira semana de vida foi de $14 \%$, não havendo diferença significativa $\quad(p>0,05)$ entre os tratamentos. A mortalidade na segunda semana de vida (dias 8 a 14) foi maior $(\mathrm{p}<0,05)$ para o feno $(7,1 \%)$ do que para maravalha $(1,7 \%)$. O consumo do feno pela coelha, mesmo após a reposição no dia 1 , pode ter diminuído a quantidade de material disponível para manutenção de temperatura dos láparos (OLIVEIRA et al., 2017). A mortalidade observada na $3^{\text {a }}$ semana foi de $2,5 \%$, não apresentando diferença significativa $(p>0,05)$ entre substratos. Não houve mortalidade de láparos na $4^{\mathrm{a}}$ e $5^{\mathrm{a}}$ semanas de vida, podendo indicar que a retirada do ninho, e consequentemente do acesso ao 
substrato, aos 21 dias, não influencia na mortalidade dos láparos próximos ao desmame (HAMILTON et al., 1997; OLIVEIRA et al., 2017). A mortalidade do nascimento ao desmame não apresentou diferença significativa $(p>0,05)$ entre os tratamentos, concordando com o descrito por Oliveira et al. (2017).

Não foi observada correlação ( $p>0,05)$ entre QNPOS e mortalidade de láparos dos dias 2 a 10 de vida, bem como entre temperatura ambiental/umidade relativa do ar e a mortalidade nesse período. Pode ser indicativo de que o comportamento dos láparos de acordo com a temperatura ambiental, ou seja, a maior cobertura em temperaturas baixas e menor em temperaturas altas, forneça a regulação necessária para a manutenção de temperatura corporal dos láparos. Outros fatores, como habilidade materna, condição corporal da coelha e tamanho do láparo, podem influenciar na mortalidade dos láparos pré-desmame (ELMAGHRABY e ELKHOLYA, 2010; TAMEEM ELDAR et al., 2012).

Foi necessária a reposição de material no D-1 em 100\% dos ninhos com feno e $50 \%$ dos ninhos com maravalha. O consumo de feno pela coelha pode explicar a maior necessidade de reposição, enquanto a de maravalha foi causado pelo ato da coelha escavar o ninho, retirando o material do seu interior. Mesmo com a reposição, a quantidade de substrato total (inicial + reposição) foi maior para os ninhos com maravalha (Tabela 5), o que é explicado pela maravalha ser um material mais compacto, sendo necessário uma maior quantidade de maravalha para chegar aos $5 \mathrm{~cm}$ de altura dentro do ninho.

Mesmo com o preço de custo similar entre os substratos estudados (feno $=\mathrm{R} \$ 0,93 / \mathrm{kg} ; \quad$ maravalha $=$ $\mathrm{R} \$ 1,00 / \mathrm{kg}$ ), a maior quantidade de substrato total necessária de maravalha fez com que esse tratamento tivesse o maior custo por ninho e por láparo desmamado. O custo do substrato de nidificação é incluído no custo fixo de serviços, junto com água, luz, telefone e manutenção, sendo que este conjunto representa $7 \%$ do custo de produção de coelhos para carne (CARTUCHE et al., 2014). Segundo Farkas et al. (2016), a maravalha pode ser utilizada como substrato de nidificação sem prejuízo produtivo. No atual estudo, o preço da maravalha teria que ser pelo menos $50 \%$ mais barato que o feno para que o custo entre ambos se equiparasse. 
Tabela 5. Quantidade de substrato (média \pm desvio padrão, g), custo por ninho e por láparo desmamado $(\mathrm{R} \$)$, de acordo com substrato

\begin{tabular}{lcc}
\hline \multicolumn{1}{c}{ Parâmetro } & Maravalha & Feno \\
\hline Quantidade total utilizada & $377,83 \pm 98,26^{\mathrm{a}}$ & $170,83 \pm 41,33^{\mathrm{b}}$ \\
por ninho (g) & $0,38 \pm 0,10^{\mathrm{a}}$ & $0,16 \pm 0,04^{\mathrm{b}}$ \\
Custo por ninho (R\$) & $0,08 \pm 0,03^{\mathrm{a}}$ & $0,04 \pm 0,01^{\mathrm{b}}$ \\
Custo por láparo &
\end{tabular}

Letras minúsculas diferentes, na mesma linha, são diferentes entre si $(\mathrm{p}<0,05)$

\section{Conclusão}

Considerando a qualidade de ninho pré e pós-parto, ganho de peso diário e mortalidade dos láparos do nascimento ao desmame, a maravalha pode ser utilizada como alternativa ao feno como material de nidificação. No entanto, a maravalha apresentou maior custo, sendo que disponibilidade e custo regional devem ser levados em consideração pelo produtor na escolha desse substrato.

\section{Agradecimentos}

Agradecimentos à PUCPR pela oportunidade e bolsa de iniciação científica PIBICJr, ao CNPq pela bolsa de iniciação científica PIBIC e ao Gustavo da Costa por todo o auxílio na coleta de dados.

\section{Referências bibliográficas}

BLUMETTO, O.; OLIVAS， I.; TORRES, A.G.; VILLAGRÁ, A. Use of straw and wood shavings as nest material in primiparous does. World Rabbit Science, v. 18, p. 237-242, 2010.

CARTUCHE， L.; PASCUAL， M.; GÓMEZ E.A.; BLASCO, A. Economic weights in rabbit meat production. World Rabbit Science, v. 22, p. 165177, 2014.

ELMAGHRABY, M.M.; ELKHOLYA, S.Z. Characterizing litter losses in purebred New Zealand White Rabbits. Lucrări Ştiinţifice- Universitatea de Ştiinţe Agricole Şi Medicină Veterinară, Seria Zootehnie, v.54, p. 304-310, 2010.

FARKAS， T.P.; SZENDRO， Z.S.; MATICS, Z.S.; RADNAI, I.; MAYER, A.; GERENCSÉR, Z.S. Effect of different nest material on performance of rabbit does. In:11th World Rabbit Congress, 2016, Qingdao, China. Proceedings ... Qingdao: WRC, 2016. p. 197-200. Acesso em 14 maio 2020. Online. Disponível em: http://worldrabbit-science.com/WRSA- 
Proceedings/Congress-2016-

Qingdao/Chine-2016-a.htm

FERREIRA W. M., MACHADOL. C., JARUCHEY. G., CARVALHOG. G., OLIVEIRAC. E. A., SOUZAJ. D. S., CARISSIMOA. P. G. Manual Prático de Cunicultura. Bambuí. ACBC, 2012.75p.

GONZÁLEZ-MARISCAL, G.; CABA, M.; $\quad$ MARTÍNEZ-GÓMEZ, $\quad$ M.; BAUTISTA, A.; HUDSON, R. Mothers and offspring: The rabbit as a model system in the study of mammalian maternal behavior and sibling interactions. Hormones and Behavior, v. 77, p.30-41, 2016.

HAMILTON, H.H.; LUKEFAHR, S.D.; MCNITT, J.I. Maternal nest quality and its influence on litter survival and weaning performance in commercial rabbits. Journal of Animal Science, v. 7, p. 926-933, 1997.

KLINGER, A.C.K.; TOLEDO, G.S.P. Cunicultura didática e prática na criação de coelhos. Santa Maria. Editora UFSM, 2018. 128p.

MACHADO, L.C.; FERREIRA, W.M. Opinião: organização e estratégias da cunicultura brasileira - buscando soluções. Revista Brasileira de Cunicultura, v.6, n. 01, p.1-31, 2014.

OLIVEIRA, M.C.; LIMA, S.C.O.; MESQUITA, A.S.; SILVA, J.Á.; GOMES, Y.S.; ATTIA, Y.A.; OLIVEIRA, H.C. Nesting material for does: effect on nest building and performance at first parturition. Revista Colombiana de Ciencias Pecuarias, v.30, p. 308-315, 2017.

ROSS. S.; ZARROW, M.X.; SAWIN, P.B.; DENENBERG, V.H.; BLUMENFIELD, M. Maternal behaviour in the rabbit under seminatural conditions. Animal Behaviour, v. 11, p.283-285, 1963.

SELTMANN, M.W.; RANGASSAMY, M.; ZAPKA, M.; HOFFMAN, K.L.; RÖDEL, H.G. Timing of maternal nest building and perinatal offspring survival in a group-living small mammal. Behavioral Ecology and Sociobiology, v.71, p. 64-76, 2017.

SZENDRO, Z.S.; TROCINO, A.; HOY, S.T.; XICCATO, G.; VILLAGRÁ, A.; MAERTENS, L. A review of recent research outcomes on the housing of farmed domestic rabbits: reproduction 
does. World Rabbit Science, v. 27, p. 114, 2019.

TAMEEM ELDAR, A.A.; ELAMIN, K.M.; YOUSIF, I.A.; HASSAN, H.E.; MUSA, A.M. Growth and mortality in pre and post-weaning rearing periods for Sudanese local rabbit. Journal of Animal Production Advances, v.2, n.5, p. 214$220,2012$.

VELLA, D.; DONNELLY, T.M. Basic anatomy, physiology and husbandry. In: QUESENBERRY, K.E.; CARPENTER, J.W. Ferrets, rabbits and rodents: clinical medicine and surgery. $3^{\mathrm{a} e d .}$ Missouri: Elsevier, 2012, p.157-173.

ZARROW, M.X.; SAWIN, P.B.; ROSS, S.; DENENBERG, V.H.; CRARY, D.; WILSON, E.D.; FAROOQ, A. Maternal behavior in the rabbit: Evidence for an endocrine basis of maternal nest building and additional data on maternal nest building in the Dutch belted race. Journal of Reproduction and Fertility, v. 2, p. 152-162, 1961. 